\title{
Cardiac resynchronization therapy is effective even in elderly patients with comorbidities
}

\author{
Natália António • Carolina Lourenço • Rogério Teixeira • Fátima Saraiva • \\ Lourenço Coelho • Miguel Ventura • João Cristóvão • Luís Elvas • Lino Gonçalves • \\ Luís A. Providência
}

Received: 24 June 2009 / Accepted: 25 September 2009 /Published online: 25 November 2009

(C) Springer Science + Business Media, LLC 2009

\begin{abstract}
Purpose The purpose of this study was to compare the effects of cardiac resynchronization therapy (CRT) in elderly patients ( $\geq 65$ years) with younger patients and to assess the impact of comorbidities in CRT remodeling response.

Methods This is a prospective study of 87 consecutive patients scheduled for CRT who underwent clinical and echocardiographic evaluation before and 6 months after CRT. A reduction in left ventricular end-systolic volume (LVESV) $\geq 15 \%$ after CRT defined remodeling responders, and a reduction of at least one New York Heart Association class defined clinical responders. Multivariate analysis was used to identify independent predictors of non-response to CRT in terms of reverse remodeling.

Results The mean age was $62 \pm 11$ years, with 36 elderly patients (41\%). The baseline QRS duration was $145 \pm$ 32 ms. After CRT, there were significant and similar improvements of left ventricular (LV) ejection fraction, LVESV, LV dP/dt, and mitral regurgitation jet area (JA) between elderly ( $\geq 65$ years) and younger ( $<65$ years) patients. The number of clinical and remodeling responders was comparable, and we found no significant differences in unplanned cardiac hospitalizations at 6 months between groups. Independent predictors of lack of remodeling response to CRT were QRS duration $<120 \mathrm{~ms}$, LV diastolic diameter $>74 \mathrm{~mm}$, and $\mathrm{JA}>10 \mathrm{~cm}^{2}$ before CRT, but not comorbidities.
\end{abstract}

N. António $(\bowtie) \cdot$ C. Lourenço $\cdot$ R. Teixeira $\cdot$ F. Saraiva $\cdot$

L. Coelho $\cdot$ M. Ventura $\cdot$ J. Cristóvão $\cdot$ L. Elvas $\cdot$ L. Gonçalves $\cdot$

L. A. Providência

Department of Cardiology,

Coimbra University Hospital and Medical School,

Avenida Bissaya Barreto,

3000-076 Coimbra, Portugal

e-mail: natalia.antonio@gmail.com
Conclusion This work suggests that being elderly is not an impediment to CRT success even in the presence of comorbidities.

Keywords Resynchronization therapy Elderly · Diabetes . Renal dysfunction $\cdot$ Hyperuricemia $\cdot$ Obesity

\section{Introduction}

Heart failure (HF) is the fastest growing cardiovascular disease, and it carries a poor prognosis even with optimal pharmacotherapy [1]. The prevalence of HF increases rapidly with age, the mean age of HF population being around 75 years in developed countries [2,3].

Cardiac resynchronization therapy (CRT) improves symptoms and also reduces HF hospitalizations and allcause mortality when added to optimal pharmacotherapy in patients in New York Heart Association (NYHA) class III or IV, with reduced ejection fraction and a QRS width of at least $120 \mathrm{~ms}$. However, in almost all randomized clinical trials, these results were demonstrated in selected patients, with few older patients enrolled [4-6]. As a result, it is still not completely clear whether elderly patients respond less favorably or not to CRT as compared with younger patients.

Conventionally, the term "elderly" has been defined as a chronological age of 65 years or older [7]. Recent studies have suggested that elderly patients derive similar benefits from CRT to younger patients [8-10]. Nevertheless, in these works, the elderly population is quite different in terms of chronological age: Foley et al. analyzed a population 80 or more years old; Bleeker et al. used 70 as cutoff, and Delnoy et al. studied a group older than 75 [8-11]. In addition, in these studies, there was no reference to comorbidities [8-10] or they were present in small rates in these older patients [11]. 
The aim of our study was to compare the effects of CRT in elderly patients ( 65 years or older) vs. younger patients (64 years or younger) in terms of clinical and echocardiographic parameters. We also aimed to assess the impact of typical heart failure-associated comorbidities in reverse remodeling and clinical response to CRT.

\section{Methods}

\subsection{Inclusion criteria and study protocol}

This is a prospective observational study that involved a single center. The study population included 87 consecutive patients with advanced HF who underwent CRT from November 2005 to October 2007. The majority of patients were in NYHA functional class III or IV despite optimal medical treatment. All patients had echocardiographic left ventricular (LV) ejection fraction $<35 \%$ and QRS duration $>120 \mathrm{~ms}$ (with left bundle branch block configuration) or QRS duration $\leq 120 \mathrm{~ms}$, but mechanical dyssynchrony documented by tissue Doppler echocardiography (intraventricular dyssynchrony $>40 \mathrm{~ms}$ and interventricular dyssynchrony $>40 \mathrm{~ms}$ ).

Patients were divided according to the chronological age into younger (less than 65 years old) and elderly patients (65 years old or older).

At baseline and 6 months after CRT, all patients underwent a clinical and echocardiographic evaluation. The clinical follow-up period was extended until 15th August 2009 (mean follow-up of $28 \pm 12$ months), with hospital file review or direct telephone contact, and we have recorded the occurrence of death, heart transplantation, and hospitalization for heart failure management.

Regarding assessed comorbidities, estimated creatinine clearance $(\mathrm{CrCl})$ was calculated with the CockcroftGault formula and renal dysfunction was defined as a $\mathrm{CrCl}<60 \mathrm{ml} / \mathrm{min}$. Hyperuricemia was defined as a serum uric acid level $>7.0 \mathrm{mg} / \mathrm{dl}$ in men or $>6.0 \mathrm{mg} / \mathrm{dl}$ in women. Patients with a body mass index of $30 \mathrm{~kg} / \mathrm{m}^{2}$ or higher were considered obese. We have also assessed history of known diabetes and persistent atrial fibrillation.

The protocol was approved by our institutional Research Ethics Committee, and all patients gave informed consent.

\subsection{Device implantation}

The LV pacing lead was inserted by a transvenous approach through the coronary sinus, with an over-the-wire system, into either a lateral or posterolateral cardiac vein whenever possible. The right atrium and right ventricle were stimulated by positioning standard bipolar leads in the right atrial appendage and right ventricular apex, respectively. A combined device (CRT plus internal defibrillator) was implanted in 64 patients (74\%).

CRT device and lead implantation was successful in all patients, without major complications.

\subsection{Echocardiography}

Standard echocardiography, including tissue Doppler imaging (TDI), was performed using an ATL IDH 5500 Philips System (Vingmed- General Electric, Horten, Norway) before and 6 months after implantation of the biventricular pacing device. LV dimensions were measured from $\mathrm{M}$ mode echocardiography in the parasternal long-axis view. The LV end-diastolic volume (LVEDV), left ventricular end-systolic volume (LVESV), and left ventricular ejection fraction (LVEF) were assessed by the biplane Simpson's equation in apical four-chamber and two-chamber views. Another measurement of systolic function evaluated was $\mathrm{dP} / \mathrm{dt}$. In fact, it has been demonstrated that the mean rate of Doppler-derived LV pressure rise during early systole (determined by the $1-$ and $3-\mathrm{m} / \mathrm{sec}$ velocity points on the rising segment of the continuous-wave mitral regurgitation curve) correlated well with peak $\mathrm{dP} / \mathrm{dt}$ obtained from $\mathrm{LV}$ pressure curves at catheterization, although $\mathrm{dP} / \mathrm{dt}$ may underestimate the true values in a substantial number of patients [12]. LV diastolic function was assessed with the evaluation of LV inflow diastolic velocities with pulsewave Doppler ( $E$ and $A$ velocities). We assessed the ratio of peak flow velocity in early diastole and peak flow velocity in late diastole during atrial contraction $(E / A)$, the deceleration time of E-wave (DTE), isovolumic relaxation time, and the mitral regurgitation jet area (JA).

To assess dyssynchrony, time delay between the onset of QRS complex on the surface electrocardiogram and the onset of the systolic velocity wave on the TDI recording was assessed in four basal LV segments (septal, lateral, anterior, and inferior). Intraventricular dyssynchrony (TS-Intra-VD) was calculated as the difference between the longest and the shortest time delay in the four basal segments. Interventricular dyssynchrony (TS-Inter-VD) was calculated as the difference between time to peak systolic velocity at the right ventricle free wall and the most delayed LV segment.

One single operator performed all echocardiographic evaluations in order to avoid inter-individual variability.

\subsection{Definition of CRT responders}

At the 6 months of follow-up, patients were classified as clinical responders to CRT if they were still alive, had not been re-hospitalized for management of congestive heart failure, and had a reduction of at least one NYHA class. Regarding echocardiographic definition of response to 
CRT, a decrease in LVESV of at least $15 \%$ defined remodeling responders [13].

\subsection{Statistical analysis}

Statistical analyses were performed using SPSS software version 15. Results are expressed as mean \pm standard deviation (SD) for continuous variables and as counts and percentages for categorical variables. Data were compared with paired or unpaired Student's $t$ test when appropriate. Nonparametric Mann-Whitney test was used for comparison of continuous variables between younger and elderly patients when variables were not normally distributed. Discrete variables were compared with the chi-square test or with Fisher exact test whichever is appropriate.

Efficacy of CRT was examined by comparing baseline vs. follow-up parameters using the Student's paired $t$ test for comparisons of quantitative variables and by the $\mathrm{McNemar}$ test for comparisons of qualitative variables.

Independent predictors of non-response to CRT were identified by means of multivariable logistic regression. The simultaneous effects of elderly and comorbidities on non-response to CRT were examined. We entered into the multivariable logistic regression model the following variables: age over 65 , renal dysfunction, diabetes, NYHA IV at baseline, left atrium $>47 \mathrm{~mm}$, QRS duration $<120 \mathrm{~ms}$, LVDD $>74 \mathrm{~mm}$, and $\mathrm{JA}>10 \mathrm{~cm}^{2}$.
The Kaplan-Meier method was used to compare heart transplantation, death, or hospitalization for heart failurefree survival between elderly and younger patients.

A $p$ value $<0.05$ was considered statistically significant.

\section{Results}

\subsection{Study population}

There were 55 male (63\%) and 32 female patients (37\%). The mean age was $62 \pm 11$ years, with 51 younger patients (59\%) and 36 elderly patients $(41 \%)$. In the overall population, hyperuricemia was present in $49 \%$, renal dysfunction in $38 \%$, diabetes in $20 \%$, obesity in $19 \%$, and atrial fibrillation in $15 \%$ of patients. Baseline characteristics of the two age groups are summarized in Table 1 . Elderly patients had a significantly higher prevalence of renal dysfunction $(69.0 \%$ vs. $19.1 \%$, $p<0.001)$ and tend to have more diabetes. They also had less frequently a baseline QRS duration $<120 \mathrm{~ms}$ ( $23.5 \%$ patients in the younger group vs. $6.1 \%$ in the elderly group, $p=0.040$ ), but LV dyssynchrony was similar in both groups (Table 1). The elderly group received less frequently a combined device ( $86 \%$ of the younger group vs. $56 \%$ of the elderly group, $p=0.003$ ). The left ventricular lead was positioned in a lateral or posterolateral vein in $73.3 \%$ of cases $(75.0 \%$ of younger vs. $66.7 \%$ of elderly patients, $p=0.645$ ).
Table 1 Comparison of baseline characteristics between elderly and younger patients
NYHA New York Heart Association, $B M I$ body mass index, $L V E F$ left ventricular ejection fraction, $L V E D V$ left ventricular end-diastolic volume, LVESV left ventricular end-systolic volume, $L V$ left ventricular, $J A$ mitral regurgitation jet area, $E / A$ E/A ratio, DTE deceleration time of E-wave

\begin{tabular}{lllr}
\hline Characteristics & $<65$ years old $(n=51)$ & $\geq 65$ years old $(n=36)$ & $p$ value \\
\hline Age & $54.7 \pm 8.3$ & $71.8 \pm 4.1$ & $<0.001$ \\
Men/women (\%) & $66.7 / 33.3$ & $58.3 / 41.7$ & 0.501 \\
NYHA class II & 11.8 & 8.3 & 0.749 \\
NYHA class III & 64.7 & 72.2 & \\
IV & $23.5 \%$ & $19.5 \%$ & \\
Ischemic/idiopathic (\%) & $33.3 / 66.7$ & $38.9 / 61.1$ & 0.653 \\
QRS duration (ms) & $141.2 \pm 33.1$ & $150.0 \pm 28.4$ & 0.212 \\
Atrial fibrillation (\%) & 12.0 & 19.4 & 0.374 \\
Diabetes (\%) & 13.7 & 29.4 & 0.077 \\
Creatinine clearance (ml/min) & $84.3 \pm 31.7$ & $55.6 \pm 19.3$ & $<0.001$ \\
BMI (kg/m ${ }^{2}$ ) & $27.5 \pm 4.1$ & $27.4 \pm 3.6$ & 0.836 \\
Uric acid (mg/dl) & $6.7 \pm 2.0$ & $6.5 \pm 2.3$ & 0.763 \\
LVEF (\%) & $23.9 \pm 6.3$ & $24.4 \pm 7.1$ & 0.746 \\
LVEDV (ml) & $278.3 \pm 118.4$ & $246.6 \pm 85.7$ & 0.174 \\
LVESV (ml) & $214.2 \pm 102.5$ & $188.2 \pm 71.7$ & 0.192 \\
LV dP/dt (mmHg/ms) & $477.5 \pm 186.6$ & $479.7 \pm 129.7$ & 0.955 \\
JA (cm ${ }^{2}$ ) & $7.9 \pm 5.9$ & $7.6 \pm 5.1$ & 0.821 \\
E/A & $1.4 \pm 0.8$ & $1.1 \pm 0.5$ & 0.097 \\
DTE (ms) & $169.6 \pm 62.5$ & $174.6 \pm 70.3$ & 0.323 \\
Intraventricular dyssynchrony (ms) & $81.7 \pm 46.2$ & $84.2 \pm 56.2$ & 0.821 \\
Interventricular dyssynchrony (ms) & $51.7 \pm 27.4$ & $47.7 \pm 21.2$ & 0.471 \\
\hline
\end{tabular}


Table 2 Echocardiographic response over the two age categories

\begin{tabular}{llc}
\hline Six-month follow-up results & Younger $(<65)$ & Elderly $(\geq 65)$ \\
\hline LV EF (\%) & & \\
Baseline & $23.9 \pm 6.3$ & $24.4 \pm 7.1$ \\
Follow-up & $31.4 \pm 9.2^{*}$ & $31.0 \pm 9.9^{*}$ \\
LVEDV (ml) & & \\
Baseline & $277.2 \pm 119.3$ & $246.6 \pm 85.7$ \\
Follow-up & $267.2 \pm 135.9$ & $228.0 \pm 92.5^{*}$ \\
LVESV (ml) & & \\
Baseline & $212.9 \pm 103.1$ & $188.2 \pm 71.7$ \\
Follow-up & $188.8 \pm 113.6^{*}$ & $161.9 \pm 79.7^{*}$ \\
JA (cm $\left.{ }^{2}\right)$ & & \\
Baseline & $8.1 \pm 5.9$ & $7.6 \pm 5.1$ \\
Follow-up & $6.2 \pm 5.1^{*}$ & $5.4 \pm 4.7^{*}$ \\
LV dyssynchrony & & \\
Baseline & $81.7 \pm 46.2$ & $84.2 \pm 56.2$ \\
Follow-up & $44.4 \pm 34.3^{*}$ & $64.9 \pm 44.9$ \\
LV dP/dt (mmHg) & & \\
Baseline & $487.4 \pm 189.7$ & $488.0 \pm 129.7$ \\
Follow-up & $667.5 \pm 298.5^{*}$ & $701.7 \pm 259.1^{*}$ \\
\hline
\end{tabular}

$L V E F$ left ventricular ejection fraction, $L V E D V$ left ventricular enddiastolic volume, LVESV left ventricular end-systolic volume, JA mitral regurgitation jet area, $L V$ left ventricular

${ }^{*} p<0.05$ (follow-up vs. baseline value)

\subsection{Effects of CRT: 6 months of follow-up}

After CRT implantation, there were significant improvements of LVEF, LVESV, LV dP/dt, and JA in both age groups (Table 2). Moreover, the magnitude of improvement of these echocardiographic parameters was not significantly different between groups. The reduction of LVESV was $24.1 \pm 43.1 \mathrm{ml}$ in the younger group vs. $26.2 \pm 52.5 \mathrm{ml}$ in the elderly group ( $p=0.838$ ); the improvement of LVEF was $7.5 \pm 8.6 \%$ in the younger group vs. $6.5 \pm 9.2 \%$ in the elderly group ( $p=0.621)$; the improvement of $\mathrm{LV} \mathrm{dP} / \mathrm{dt}$ was $180.2 \pm 348.6 \mathrm{mmHg} / \mathrm{ms}$ in the younger vs. $217.7 \pm 269.1 \mathrm{mmHg} / \mathrm{ms}$ in the elderly group $(p=0.630)$; and reduction of JA was $1.8 \pm 3.2 \mathrm{~cm}^{2}$ in the younger vs. $2.3 \pm 4.0 \mathrm{~cm}^{2}$ in the elderly group $(p=0.589$ ).

The number of remodeling responders and of clinical responders was comparable between younger and elderly patients (Fig. 1). During the 6-month follow-up period, we found no significant differences either in heart failure-driven rehospitalization or in ICD shocks between groups (Fig. 1). Only one patient underwent cardiac transplantation during the follow-up, and another one died due to heart failure.

\subsection{Predictors of non-response to CRT}

In the overall population, the proportion of remodeling nonresponders to CRT was $46 \%$. No significant differences between remodeling responders and non-responders to CRT were found regarding age, the incidence of diabetes, renal dysfunction, hyperuricemia, obesity, and the presence of ischemic cardiomyopathy. However, non-responders were more frequently in NYHA class IV before CRT (32.5\% vs. $13 \%$ in remodeling responders, $p=0.038$ ), presented more frequently a QRS duration less than $120 \mathrm{~ms}(28 \% \mathrm{vs} .7 \%$, $p=0.009$ ), with significantly larger baseline left atrium, higher LV dimensions and volumes, and greater mitral regurgitation JA than remodeling responders (Table 3).

In multivariate logistic regression analysis, independent predictors of non-response to CRT were baseline QRS duration less than $120 \mathrm{~ms}$, LVDD higher than $74 \mathrm{~mm}$, and JA over $10 \mathrm{~cm}^{2}$ before CRT (Fig. 2). Comorbidities were not independent predictors of remodeling non-response to CRT.

In elderly patients, CRT response in terms of reverse remodeling was correlated to $E / A$ ratio, E-wave deceleration time, LV dyssynchrony, and QRS duration. The E/A ratio was inversely correlated with the reduction of LVESV ( $r=-0.602, p=0.001)$. E-wave deceleration time, LV dyssynchrony, and QRS duration were directly correlated with LVESV reduction after CRT $(r=0.377, p=0.026 ; r=0.418$, $p=0.007$; and $r=0.378, p=0.021$, respectively).

None of the studied comorbidities was correlated with reverse remodeling after CRT in the elderly group.

\subsection{Extended clinical follow-up}

In the extended follow-up, the occurrence of heart transplantation, hospitalization for heart failure management, or death was verified in $27.6 \%$ of patients, with a mean time to this event of $14 \pm 10$ months. The Kaplan-Meier survival analysis revealed no significant differences in event-free survival curves between the elderly and the younger groups (Fig. 3).

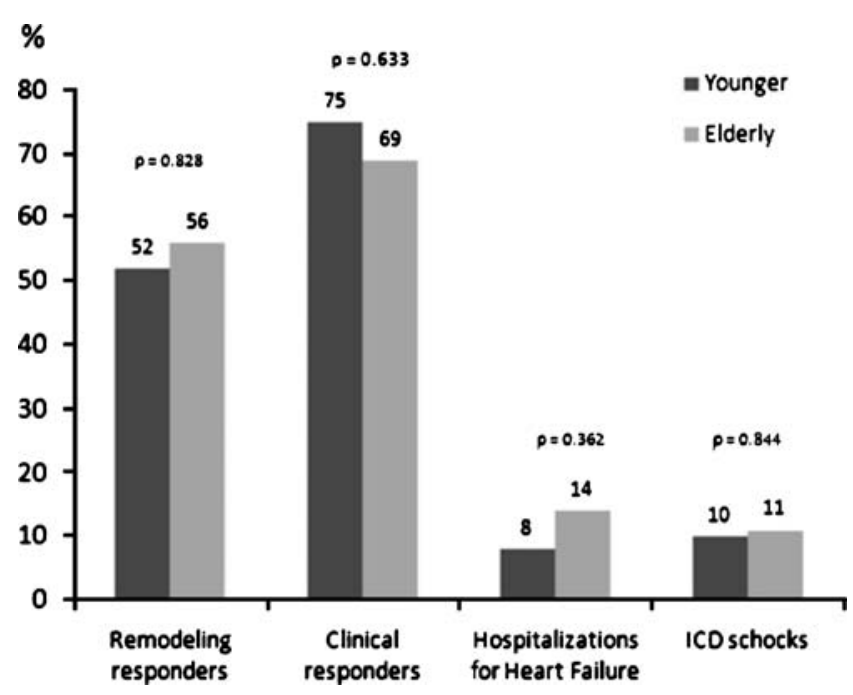

Fig. 1 Clinical outcomes over the different age categories 
Table 3 Baseline characteristics of remodeling responders and nonresponders to cardiac resynchronization therapy

\begin{tabular}{lllr}
\hline & Responders & Non-responders & $p$ value \\
\hline Age & $63 \pm 11$ & $61 \pm 11$ & 0.360 \\
Men/women (\%) & $57 / 43$ & $73 / 27$ & 0.090 \\
Baseline NYHA class (\%) & & & \\
II & 15 & 5 & 0.050 \\
III & 72 & 62 & \\
IV & 13 & 33 & \\
QRS duration (ms) & $150 \pm 30$ & $139 \pm 33$ & 0.110 \\
Atrial fibrillation (\%) & 17.8 & 12.5 & 0.559 \\
Ischemic/idiopathic (\%) & $34.8 / 65.2$ & $35.0 / 65.0$ & 0.983 \\
Diabetes (\%) & 23.9 & 15.8 & 0.356 \\
Renal dysfunction (\%) & 36.6 & 41.2 & 0.684 \\
Obesity (\%) & 22.2 & 12.8 & 0.392 \\
Hyperuricemia (\%) & 53.1 & 43.8 & 0.453 \\
Left atrium (mm) & $43 \pm 9$ & $47 \pm 8$ & 0.020 \\
LVDD (mm) & $71 \pm 7$ & $79 \pm 11$ & $<0.001$ \\
LVSD (mm) & $59 \pm 7$ & $67 \pm 12$ & 0.001 \\
LVEDV (ml) & $237 \pm 73$ & $296 \pm 130$ & 0.009 \\
LVESV (ml) & $181 \pm 63$ & $227 \pm 112$ & 0.020 \\
LVEF (\%) & $28 \pm 10$ & $24 \pm 6$ & 0.168 \\
JA (cm ${ }^{2}$ ) & $6.0 \pm 4.8$ & $9.9 \pm 5.8$ & 0.001 \\
LV dyssynchrony (ms) & $84.5 \pm 57.6$ & $80.4 \pm 41.7$ & 0.710 \\
\hline
\end{tabular}

NYHA New York Heart Association, $L V D D$ left ventricular diastolic diameter, $L V S D$ left ventricular systolic diameter, $L V E D V$ left ventricular end-diastolic volume, $L V E S V$ left ventricular end-systolic volume, $L V E F$ left ventricular ejection fraction, $J A$ mitral regurgitation jet area, $L V$ left ventricular

\section{Discussion}

Despite the encouraging results from CRT in recent trials, patients' responses to CRT vary significantly. Presently, it remains unclear whether or not elderly patients, of the real

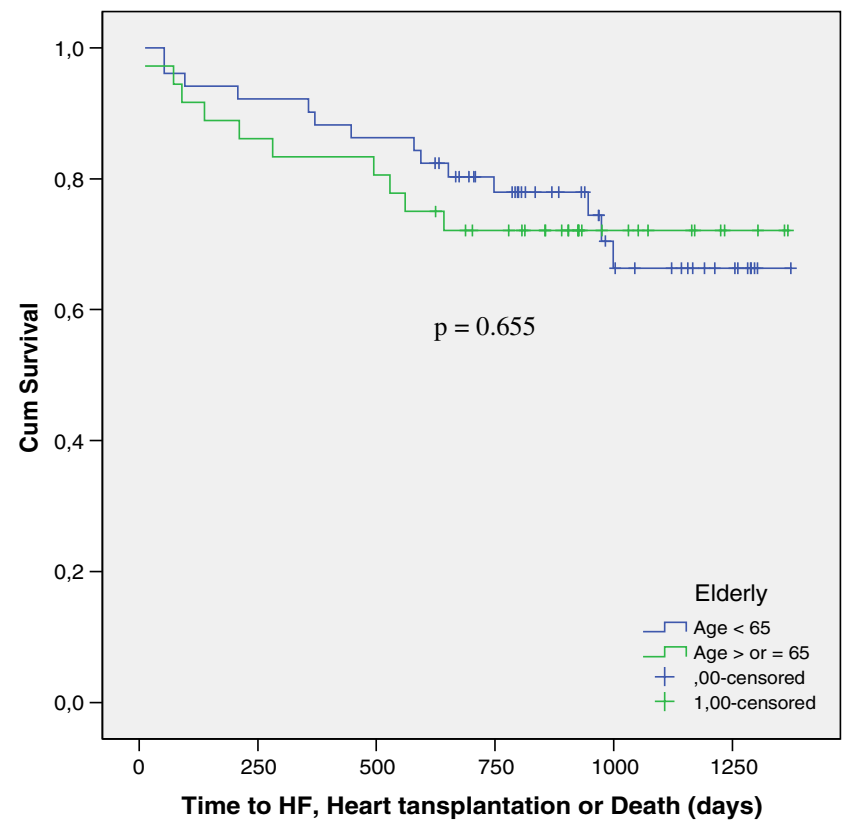

Fig. 3 Kaplan-Meier event-free survival of patients aged $\geq 65$ and patients aged $<65$ years

world, respond less favorably to CRT as compared with younger patients. If age was a negative influence on clinical outcome, this would be a good reason to restrict the use of CRT in the elderly.

Previous studies have shown that elderly patients with HF are a heterogeneous group and appear to differ substantially from patients enrolled in clinical trials [14]. Comorbidity complicates HF care and is prevalent in one form or another for the majority of elderly patients scheduled for CRT. This wide range of comorbidities, which includes diabetes and renal dysfunction, contributes to the progression of $\mathrm{HF}$ and may alter the response to treatment [15-17].

Previous data have demonstrated that the number and severity of comorbid illnesses is a key factor in the overall
Fig. 2 Multivariate logistic regression analysis of predictors of non-response to cardiac resynchronization therapy
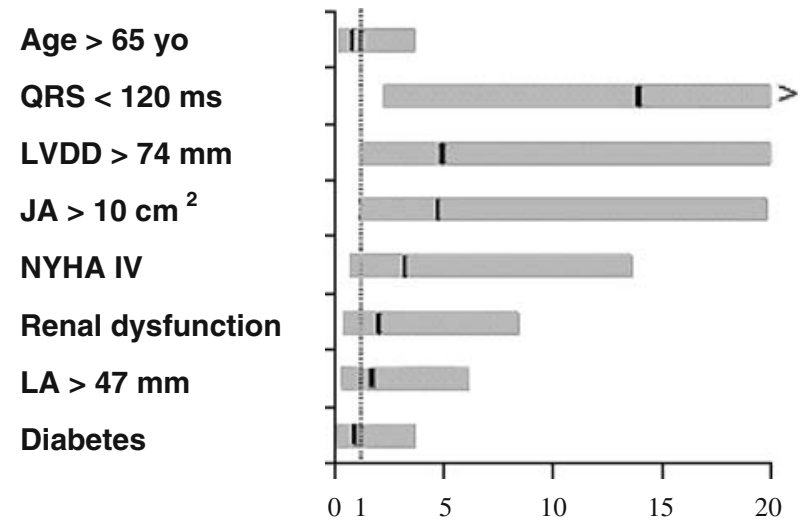

$\begin{array}{lcc}\text { OR } & \text { CI } 95 \% & p \text { value } \\ 0.79 & 0.184-3.375 & 0.749 \\ 13.80 & 2.266-84.036 & 0.004 \\ 4.83 & 1.288-18.142 & 0.020 \\ 4.62 & 1.090-19.553 & 0.038 \\ 3.11 & 0.723-13.359 & 0.128 \\ 1.89 & 0.440-8.127 & 0.391 \\ 1.61 & 0.441-5.847 & 0.473 \\ 0.79 & 0.178-3.510 & 0.758\end{array}$


survival of patients as well as the benefits of therapy [18]. So, we could ask: could comorbidities be an impediment to clinical and echocardiographic response to CRT?

In "the real world" clinical practice, elderly patients referred to CRT usually present comorbidities that distinguish them from the perfect candidate enrolled in clinical trials. Previous studies have looked at the impact of individual comorbidities or composite scores on mortality after CRT rather than their impact on CRT response [19, 20]. In fact, there are no studies that have examined the impact of comorbidities such as diabetes, renal dysfunction, obesity, and hyperuricemia on echocardiographic response to CRT, particularly in elderly patients. To the best of our knowledge, we were the first to do so.

\subsection{Patient population}

Several baseline characteristics of our study population were consistent with those of patients enrolled in previous CRT clinical trials, such as a predominance of male gender and NYHA functional class III, a marked systolic dysfunction as revealed by reduced LVEF, a severe LV dilatation, and a prolonged QRS duration. However, in our "real world" population, we had more patients over 65 years old than in previously published clinical trials [4, 6, 21-23].

In the elderly group, we observed several comorbidities that suggest that elderly patients were not selected on the basis of a special good clinical status.

\subsection{Clinical outcome}

At the 6-month follow-up visit, CRT resulted in significant benefits in both age categories, as evidenced by the improvement in clinical and echocardiographic parameters. CRT resulted in a significant improvement in LVEF and a left ventricular reverse remodeling. Moreover, the number of clinical and remodeling responders to CRT was similar in both groups, and there were no significant differences in unplanned cardiac hospitalizations within 6 months of CRT, thus supporting the hypothesis of an inducible favorable clinical effect and reverse remodeling even in older patients.

\subsection{Impact of comorbidities}

To the best of our knowledge, no data about the impact of comorbidities like renal dysfunction, obesity, and hyperuricemia in CRT response have been reported before.

One of the most important comorbidities in HF is renal dysfunction, and it is an important independent predictor of mortality and morbidity among this population [16]. Previous studies have indicated that CRT can be considered a renal-protective strategy in $\mathrm{HF}$ and that the improvement in renal function can be another mechanism to explain the beneficial effects of CRT [24-26]. Nevertheless, there are no studies in the literature that addressed the impact of renal dysfunction on the efficacy of CRT in terms of left ventricular reverse remodeling.

In our study, renal dysfunction tended to be slightly more prevalent in non-responders. However, in multivariate analysis, it was not a predictor of non-response to CRT. Even in elderly patients who had a higher prevalence of renal dysfunction, it seems not to prevent the positive response to CRT.

Heart failure and diabetes are strongly associated, and each condition represents a risk factor for the development of the other. Additionally, the presence of diabetes is a powerful independent predictor of morbidity and mortality among patients with HF [27]. The pathophysiology underlying HF in diabetic patients differs from that of non-diabetic patients, and it has been questioned whether CRT is equally beneficial in patients with and without diabetes. In accordance with previous studies, in our work, diabetes was not a predictor of poor response to CRT $[28,29]$.

Serum uric acid is intensively correlated with circulating markers of inflammation in patients with chronic HF and is a strong independent predictor of mortality. Inflammatory markers reflect HF severity, so we could speculate that high uric acid levels could be associated with a poor response to CRT. However, in our population, the proportion of hyperuricemic patients in remodeling responders was similar or even higher than that of non-responders.

Obesity represents an important risk factor for overall mortality and for development of HF in the general population [30]. Nevertheless, in patients with chronic HF, obesity appears to be associated with a favorable prognosis [31-34]. The underlying pathophysiology of this "obesity paradox" is still poorly understood. In the CRT setting, no previous studies have examined whether obesity affects or not the ability of left ventricular reverse remodeling. Our study is the first to do so, and it suggests that obesity is not an impediment to CRT positive response.

A recent meta-analysis of prospective cohort studies concluded that patients in atrial fibrillation show significant improvement after CRT, with similar or improved ejection fraction as sinus rhythm patients [35]. In accordance with previous study, in our work, atrial fibrillation did not appear to be a predictor of non-response to CRT.

In our work, comorbidities were not predictors of poor response to CRT, neither in the overall population nor in elderly patients.

\subsection{Predictors of non-response to CRT}

In our study, we included some patients with narrow QRS and mechanical dyssynchrony documented by echocardiography because at the beginning of this study, the results of the RethinQ study, providing evidence that patients with 
HF and narrow QRS intervals may not benefit from CRT, were not yet known [36]. In our work, the stronger predictor of non-response to CRT was QRS less than $120 \mathrm{~ms}$, which is in accordance with the results of the RethinQ study and suggests that CRT should not be offered to patients with a narrow QRS even if they present significant echocardiographic intraventricular dyssynchrony. LV dilatation and severe mitral regurgitation were the other predictors of lack of response, underlying the importance of LV geometry in reverse remodeling after CRT. It seems that patients with advanced forms of dilated cardiomyopathy are worse candidates for CRT than patients in the earlier phases of the disease.

In the elderly, mechanical dyssynchrony, again, seems to be crucial to a positive CRT response. In addition, in these patients, parameters of diastolic function were important factors for reverse remodeling. In our study, higher $E / A$ ratios were associated with poor response to CRT, emphasizing the importance of cardiac compliance to achieve reverse remodeling.

\subsection{Study limitations}

This is a single-center, observational cohort study. The most important limitations of this study are the small number of patients included and the relatively short period of follow-up. Additionally, this work presents typical limitations of nonrandomized studies that are a potential bias in patient's selection.

Another limitation of this study is the classification of diabetes that was made on the basis of medical history obtained from patients during the baseline visit interview; information on diabetic medications or diabetes control was not collected. Moreover, we did not ascertain cases of diabetes that developed during the study follow-up.

\subsection{Clinical implications}

Our work indicates that old age is not a reason for excluding patients from CRT. In fact, our study suggests that even elderly patients with comorbidities, but with good life expectancy, can benefit from CRT.

Acknowledgments The authors thank the Portuguese Society of Cardiology/CNCDC (Centro Nacional de Colecção de Dados em Cardiologia) for their guidance and suggestions on statistical analyses.

\section{References}

1. McAlister, F. A., Tu, J. V., Newman, A., Lee, D. S., Kimber, S., Cujec, B., et al. (2006). How many patients with heart failure are eligible for cardiac resynchronization? Insights from two prospective cohorts. European Heart Journal, 27(3), 323-329.
2. Swedberg, K., Cleland, J., Dargie, H., Drexler, H., Follath, F., Komajda, M., et al. (2005). Guidelines for the diagnosis and treatment of chronic heart failure: executive summary (update 2005): The Task Force for the Diagnosis and Treatment of Chronic Heart Failure of the European Society of Cardiology. European Heart Journal, 26(11), 1115-1140.

3. Task Force for Diagnosis and Treatment of Acute and Chronic Heart Failure 2008 of European Society of Cardiology, Dickstein, K., Cohen-Solal, A., Filippatos, G., McMurray, J. J., Ponikowski, P., et al. (2008). ESC guidelines for the diagnosis and treatment of acute and chronic heart failure 2008: The Task Force for the Diagnosis and Treatment of Acute and Chronic Heart Failure 2008 of the European Society of Cardiology. European Heart Journal, 29(19), 2388-2442. (developed in collaboration with the Heart Failure Association of the ESC (HFA) and endorsed by the European Society of Intensive Care Medicine (ESICM)).

4. Abraham, W. T., Fisher, W. G., Smith, A. L., Delurgio, D. B., Leon, A. R., Loh, E., et al. (2002). Cardiac resynchronization in chronic heart failure. New England Journal of Medicine, 346(24), 1845-1853.

5. Linde, C., Leclercq, C., Rex, S., Garrigue, S., Lavergne, T., Cazeau, S., et al. (2002). Long-term benefits of biventricular pacing in congestive heart failure: results from the MUltisite STimulation in cardiomyopathy (MUSTIC) Study. Journal of the American College of Cardiology, 40(1), 111-118.

6. Cleland, J. G., Daubert, J. C., Erdmann, E., Freemantle, N., Gras, D., Kappenberger, L., et al. (2005). The effect of cardiac resynchronization on morbidity and mortality in heart failure. New England Journal of Medicine, 352(15), 1539-1549.

7. Orimo, H., Ito, H., Suzuki, T., Araki, A., Hosoi, T., \& Sawabe, M. R. (2006). Reviewing the definition of "elderly". Geriatrics \& Gerontology International, 6, 149-158.

8. Bleeker, G. B., Schalij, M. J., Molhoek, S. G., Boersma, E., Steendijk, P., van Der Wall, E. E., et al. (2005). Comparison of effectiveness of cardiac resynchronization therapy in patients $<70$ versus $\geq 70$ years of age. American Journal of Cardiology, 96(3), 420-422.

9. Delnoy, P. P., Ottervanger, J. P., Luttikhuis, H. O., Elvan, A., Misier, A. R., Beukema, W. P., et al. (2008). Clinical response of cardiac resynchronization therapy in the elderly. American Heart Journal, 155(4), 746-751.

10. Foley, P. W., Chalil, S., Khadjooi, K., Smith, R. E., Frenneaux, M. P., \& Leyva, F. (2008). Long-term effects of cardiac resynchronization therapy in octogenarians: A comparative study with a younger population. Europace, 10(11), 1302-1307.

11. Achilli, A., Turreni, F., Gasparini, M., Lunati, M., Sassara, M., Santini, M., et al. (2007). Efficacy of cardiac resynchronization therapy in very old patients: The Insync/Insync ICD Italian Registry. Europace, 9(9), 732-738.

12. Bargiggia, G. S., Bertucci, C., Recusani, F., Raisaro, A., de Servi, S., Valdes-Cruz, L. M., et al. (1989). A new method for estimating left ventricular $\mathrm{dP} / \mathrm{dt}$ by continuous wave Doppler-echocardiography. Validation studies at cardiac catheterization. Circulation, 80(5), 1287-1292.

13. Yu, C. M., Fung, J. W., Zhang, Q., Chan, C. K., Chan, Y. S., Lin, H., et al. (2004). Tissue Doppler imaging is superior to strain rate imaging and postsystolic shortening on the prediction of reverse remodeling in both ischemic and nonischemic heart failure after cardiac resynchronization therapy. Circulation, 110(1), 66-73.

14. Edward, P. H., Frederick, A. M., Kelly, A. W., Pam, W., Diana, L. O., \& Harlan, M. K. (2002). Spectrum of heart failure in older patients: Results from the national heart failure project. American Heart Journal, 143(3), 412-417.

15. Lang, C., \& Mancini, D. (2007). Non-cardiac comorbidities in chronic heart failure. Heart, 93(6), 665-671.

16. Liu, P. (2008). Cardiorenal syndrome in heart failure: A cardiologist's perspective. Canadian Journal of Cardiology, 24 (B), 25B-29B. 
17. González-Juanatey, J., Alegría Ezquerra, E., Bertoméu Martínez, V., Conthe Gutiérrez, P., de Santiago Nocito, A., \& Zsolt Fradera, I. (2008). Heart failure in outpatients: Comorbidities and management by different specialists. The EPISERVE Study. Revista Espanola de Cardiologia, 61(6), 611-619.

18. Charlson, M., Pompei, P., Ales, K., \& MacKenzie, C. (1987). A new method of classifying prognostic comorbidity in longitudinal studies: Development and validation. Journal of Chronic Diseases, 40(5), 373-383.

19. Bai, R., Di Biase, L., Elayi, C., Ching, C. K., Barrett, C., Philipps, K., et al. (2008). Mortality of heart failure patients after cardiac resynchronization therapy: Identification of predictors. Journal of Cardiovascular Electrophysiology, 19(12), 1259-1265.

20. Shalaby, A., El-Saed, A., Voigt, A., Albany, C., \& Saba, S. (2008). Elevated serum creatinine at baseline predicts poor outcome in patients receiving cardiac resynchronization therapy. Pacing and Clinical Electrophysiology, 31(5), 575-579.

21. Cazeau, S., Leclercq, C., Lavergne, T., Walker, S., Varma, C., Linde, C., et al. (2001). Effects of multisite biventricular pacing in patients with heart failure and intraventricular conduction delay. New England Journal of Medicine, 344(12), 873-880.

22. Bristow, M. R., Saxon, L. A., Boehmer, J., Krueger, S., Kass, D. A., De Marco, T., et al. (2004). The comparison of medical therapy, pacing, and defibrillation in heart failure I. Cardiacresynchronization therapy with or without an implantable defibrillator in advanced chronic heart failure. New England Journal of Medicine, 350(21), 2140-2150.

23. Cleland, J. G., Daubert, J. C., Erdmann, E., Freemantle, N., Gras, D., Kappenberger, L., et al. (2006). Longer-term effects of cardiac resynchronization therapy on mortality in heart failure [the CArdiac REsynchronization-Heart Failure (CARE-HF) Trial extension phase]. European Heart Journal, 27(16), 1928-1932.

24. Boerrigter, G., Costello-Boerrigter, L., Abraham, W., Sutton, M., Heublein, D., Kruger, K., et al. (2008). Cardiac resynchronization therapy improves renal function in human heart failure with reduced glomerular filtration rate. Journal of Cardiac Failure, 14(7), 539-546.

25. Fung, J., Szeto, C., Chan, J., Zhang, Q., Chan, H., Yip, G., et al. (2007). Prognostic value of renal function in patients with cardiac resynchronization therapy. International Journal of Cardiology, 122(1), 10-16.

26. Kimura, S., Ito, M., Chinushi, M., Tanaka, K., Tanabe, Y., Hosaka, Y., et al. (2008). Preservation of renal function in response to cardiac resynchronization therapy. Circulation Journal, 72(11), 1794-1799.

27. Ghali, J., Boehmer, J., Feldman, A., Saxon, L., Demarco, T., Carson, P., et al. (2007). Influence of diabetes on cardiac resynchronization therapy with or without defibrillator in patients with advanced heart failure. Journal of Cardiac Failure, 13(9), 769-773.

28. Kiès, P., Bax, J. J., Molhoek, S. G., Bleeker, G. B., Boersma, E., Steendijk, P., et al. (2005). Comparison of effectiveness of cardiac resynchronization therapy in patients with versus without diabetes mellitus. American Journal of Cardiology, 96(1), 108-111.

29. Fantoni, C., Regoli, F., Ghanem, A., Raffa, S., Klersy, C., Sorgente, A., et al. (2008). Long-term outcome in diabetic heart failure patients treated with cardiac resynchronization therapy. European Journal of Heart Failure, 10(3), 298-307.

30. Calle, E. E., Thun, M. J., Petrelli, J. M., Rodriguez, C., \& Heath, C. W. (1999). Body-mass index and mortality in a prospective cohort of U.S. adults. New England Journal of Medicine, 341(15), 1097-1105.

31. Cicoira, M., Maggioni, A. P., Latini, R., Barlera, S., Carretta, E., Janosi, A., et al. (2007). Body mass index, prognosis and mode of death in chronic heart failure: Results from the Valsartan Heart Failure Trial. European Journal of Heart Failure, 9(4), 397-402.

32. Bozkurt, B., \& Deswal, A. (2005). Obesity as a prognostic factor in chronic symptomatic heart failure. American Heart Journal, 150(6), 1233-1239.

33. Horwich, T. B., Fonarow, G. C., Hamilton, M. A., MacLellan, W. R., Woo, M. A., \& Tillisch, J. H. (2001). The relationship between obesity and mortality in patients with heart failure. Journal of the American College of Cardiology, 38(3), 789-795.

34. Oreopoulos, A., Padwal, R., Kalantar-Zadeh, K., Fonarow, G. C., Norris, C. M., \& McAlister, F. A. (2008). Body mass index and mortality in heart failure: A meta-analysis. American Heart Journal, 156(1), 13-22.

35. Upadhyay, G. A., Choudhry, N. K., Auricchio, A., Ruskin, J., \& Singh, J. P. (2008). Cardiac resynchronization in patients with atrial fibrillation: A meta-analysis of prospective cohort studies. Journal of the American College of Cardiology, 52(15), 1239-1246.

36. Beshai, J. F., Grimm, R. A., Nagueh, S. F., Baker, J. H., II, Beau, S. L., Greenberg, S. M., et al. (2007). Cardiac-resynchronization therapy in heart failure with narrow QRS complexes. New England Journal of Medicine, 357(24), 2461-2471. 